\title{
MicroRNA-633 enhances melanoma cell proliferation and migration by suppressing KAI1
}

\author{
ZHENGXIANG WANG $^{1}$ and YALING LIU $^{2}$ \\ ${ }^{1}$ Department of Dermatology, Hebei Medical University, Shijiazhuang, Hebei 050030; \\ ${ }^{2}$ Department of Dermatology, The Third Hospital of Hebei Medical University, Shijiazhuang, Hebei 050031, P.R. China
}

Received July 24, 2020; Accepted November 17, 2020

DOI: $10.3892 / \mathrm{ol} .2020 .12349$

\begin{abstract}
The aim of the present study was to determine the impact of microRNA (miRNA/miR)-633 on the biological properties of malignant melanoma cells. Kang-Ai 1 (KAI1), also known as cluster of differentiation 82, is an important transcriptional regulator and tumor suppressor gene present in different types of tumors. miRNAs that potentially bind with KAI1 were predicted via bioinformatics analyses. In total, six putative miRNA regulators of KAI1 were identified in the present analysis, among which miR-633 was upregulated the most in melanoma tissues compared with the control group. The expression levels of miR-633 and KAI1 in melanoma tissues compared with adjacent normal tissues were then assessed. It was found that miR-633 was significantly upregulated in melanoma cells compared with the control group, whereas the expression levels of KAI1 showed the opposite results. miR-633 was predicted to target the 3'-untranslated region of KAI1 using predictive online tools, and results from luciferase reporter assays confirmed the direct regulation of KAI1 promoter activity by miR-633. Furthermore, miR-633 mimics over expression was shown to suppress both mRNA and protein expression of KAI1, while miR-633 inhibition resulted in decreased viability and migrationin melanoma cells in vitro. Taken together, the present study demonstrated, to the best of the authors' knowledge for the first time, that miR-633 exerts an important role in melanoma through targeting KAI1.
\end{abstract}

\section{Introduction}

Melanoma is one of the most commonly occurring forms of cancer, and malignant melanoma is the third most common skin malignancy (1-6). For most patients with melanoma, immunotherapy, chemotherapy or small-molecule inhibitor

Correspondence to: Professor Yaling Liu, Department of Dermatology, The Third Hospital of Hebei Medical University, 139 Ziqiang Road, Shijiazhuang, Hebei 050031, P.R. China

E-mail: yzling_liu@126.com

Key words: melanoma, CD82 antigen, microRNA-633, bioinformatics, migration administration are not effective therapies (7). Furthermore, the outcomes of patients with advanced-stage disease remain poor $(8,9)$.

MicroRNAs (miRNAs/miRs) are short RNAs ( 22 nucleotides in length) that do not encode protein, and yet are important regulators of oncogenesis $(10,11)$. By binding to complementary sequences in the 3'-untranslated region (3'-UTR) of target mRNAs, miRNAs can alter the stability and translation of these transcripts, thereby influencing phenotypic outcomes within cells. A single miRNA can target multiple different mRNAs, giving rise to complex regulatory networks that control diverse pathological and physiological biological activities $(12,13)$. The dysregulation of miRNAs is a common hallmark of oncogenesis and tumor progression $(14,15)$. By evaluating patterns of miRNA expression, it may be possible to better diagnose or monitor specific types of cancer. For example, miR-144 and miR-92a have been shown to serve as valuable and specific diagnostic biomarkers that can guide the detection of specific subtypes of colorectal cancer (16). Additionally, miR-221 can suppress the expression of PHD finger protein 2, thereby influencing liver cancer invasion, leading researchers to highlight this signaling axis as a potential target for therapeutic intervention (17). Efforts have also been made to successfully identify patterns of miRNA dysregulation associated with melanoma (18). Given their essential roles as regulators of cancer onset and progression, further analyses of miRNAs in these oncogenic contexts are warranted.

Tetraspanin cluster of differentiation 82(CD82), also known as Kang-Ai 1 (KAI1), is an important tumor suppressor gene that was first detected based upon analyses of human metastatic prostate cancer samples (19). There is robust evidence linking KAI1 downregulation with the invasive and metastatic activities of various tumors based upon histopathological and molecular analyses (20). KAI1 mutations and associated loss-of-function are evident in a range of tumor types, reaffirming the role of this gene as a tumor suppressor (21-23). KAI1 expression in melanoma has been shown to be associated with tumor grade and patient prognosis, and it has been validated as a risk factor for disease progression $(24,25)$. However, the association between miR-633 and malignant melanoma has not been elucidated to date. The present study investigated the role of miR-633 in the proliferation and migration of melanoma cells. Furthermore, the potential role of miR-633 in regulating KAI1 expression in melanoma cells was explored. 


\section{Materials and methods}

Prediction of the candidate miRNA associated with KAIl. The online target gene prediction databases TargetScan (http://http://www.targetscan.org/vert_72), StarBase (http://starbase.sysu.edu.cn) and miRanda (http://www. microrna.orgmicrorna) were used to identify miRNAs that could be associated with KAI1, and to predict the binding region of miRNA-633 to the 3'-UTR of KAI1.

Sample collection. All cancer tissues $(\mathrm{n}=11)$ and paracancerous tissues $(n=10)$ used in the present study were collected via surgical resection from patients with melanoma at Cangzhou Central Hospital (Table I). No patients received any treatment, including chemotherapy or radiation therapy, prior to surgery. According to the melanoma treatment guidelines, the resection range was determined based on the different stage, and the paracancerous tissues were taken as close to the outer edge as possible to ensure that it was the adjacent normal tissue. Resected samples were subjected to pathological confirmation, after which they were snap-frozen with liquid nitrogen and stored at $-80^{\circ} \mathrm{C}$. However, tumor cells were present at the outer edge in one of the patients, sol1 cancer tissues and 10 paracancerous tissues were used in the present study. Patients and their families were informed of all study protocols and were asked to sign informed consent forms. The Ethics Committee of Cangzhou Central Hospital approved the present study.

Cell culture and transfection. Normal human primary epidermal melanocytes (HeMn); (The Cell Bank of Type Culture Collection of Chinese Academy of Sciences) were maintained in F-12K medium(Gibco; Thermo Fisher Scientific, Inc.) supplemented with 10\% FBS (Gibco; Thermo Fisher Scientific, Inc.). A375, A2058, B16, MEL-RM and M21 melanoma cell lines(The Cell Bank of Type Culture Collection of Chinese Academy of Sciences) were maintained in RPMI-1640 media supplemented with $10 \%$ fetal bovine serum (both purchased from Gibco; Thermo Fisher Scientific, Inc.). All cells were grown in humidified $5 \% \mathrm{CO}_{2}$ incubators at $37^{\circ} \mathrm{C}$. A375 and B16 cells were co-transfected with KAI1 wild-type (WT) or KAI1 mutant (MUT), and/or with miR-633 inhibitors or inhibitor-negative control (NC) and miR-633 mimics or mimic-NC. The transfection of cells was performed with the plasmids (Promega Corporation) using Lipofectamine ${ }^{\circledR} 3000$ (Invitrogen; Thermo Fisher Scientific, Inc.) in accordance with the manufacturer's protocol, with downstream analyses conducted at 24-48 h post-transfection. The sequences of oligo-ribonucleotides were as follows: miR-633 mimics, 5'-CUAAUAGUAUCUACCACAAUA AA-3'; mimic-NC 5'-UUCUCCGAACGUGUCACGUTT-3'; miR-633 inhibitor, 5'-UUUAUUGUGGUAGAUACUAUU AG-3'; and inhibitor-NC, 5'-CAGUACUUUUGUGUAGUA CAA-3'.

Reverse transcription-quantitative PCR (RT-qPCR). TRIzol ${ }^{\circledR}$ reagent (Invitrogen; Thermo Fisher Scientific, Inc.) was used to extract total RNA from the cells and tissues, after which reverse transcription was performed with a PrimeScript RT reagent kit (Takara Biotechnology Co., Ltd.). After the cDNA concentrations were quantified, qPCR was conducted with SYBR ${ }^{\circledR}$
Table I. Clinicopathological characteristics of patients with melanoma $(n=10)$.

\begin{tabular}{lc}
\hline Characteristic & Number of patients, $\mathrm{n}$ \\
\hline Total & 10 \\
Age, years & \\
$<45$ & 2 \\
$\geq 45$ & 8 \\
Sex & \\
Male & 7 \\
Female & 3 \\
TNM classification & \\
I+II & 7 \\
III+IV & 3 \\
Distant metastasis & \\
No & 9 \\
Yes & 1 \\
\hline
\end{tabular}

TNM, tumor-metastasis-node.

Premix Ex Taq (Takara Biotechnology Co., Ltd.) following the manufacturer's instructions. GAPDH and U6 were utilized as normalization controls for mRNA and miRNA levels, respectively. Relative fold changes in expression levels were calculated using the $2^{-\triangle \Delta C \mathrm{q}}$ method (26). Primers used were as follows: KAI1, forward 5'-ATCCGATATCCGATCGACATGAGA GGAGTTCGAT-3' and reverse 5'-CTAGGCGAGATAGAC TACCATG-3'; GAPDH, forward 5'-ATCCGATTACCGATA CCTAGACC-3' and reverse 5'-ATGGACTATATCCGACGA CGA-3'; miR-633, forward 5'-CCGATACGATGAGAGAAA CCCTGA-3' and reverse 5'-GGACAGAGTTGACTTAAG GCTAGA-3'; and U6, forward 5'-TGCGTTCCCTTTGTCATC CT-3' and reverse, 5'-AACGCTTCAC-GAATTTGCGT-3'. The thermocycling conditions were as follows: $95^{\circ} \mathrm{C}$ for $30 \mathrm{sec}$, followed by 40 cycles of $92^{\circ} \mathrm{C}$ for $5 \mathrm{sec}$ and $60^{\circ} \mathrm{C}$ for $30 \mathrm{sec}$, dissociation at $60^{\circ} \mathrm{C}$ for $1 \mathrm{~min}$ and $95^{\circ} \mathrm{C}$ for $1 \mathrm{sec}$.

Dual-luciferase reporter assay. Cells were transfected with KAI1WT or KAI1MUT luciferase reporter plasmids (Promega Corporation), along with miR-633 mimics or control constructs for $24 \mathrm{~h}$ at $37^{\circ} \mathrm{C}$ using Lipofectamine ${ }^{\circledR} 3000$ reagent (Invitrogen; Thermo Fisher Scientific, Inc.) in accordance with the manufacturer's protocol. Then, a dual-luciferase reporter assay system (Promega Corporation) was utilized to quantify luciferase activity. Renilla luciferase activity was used as the internal control, and data are presented as the ratio of firefly to Renilla luciferase activities.

Cell Counting Kit-8 (CCK-8) assay. Cells were added to 96 -well plates $\left(2 \times 10^{3}\right.$ cells/well). At the indicated time points, $10 \mu \mathrm{l}$ CCK-8 solution (Dojindo Molecular Technologies, Inc.) was added per well, and absorbance at $450 \mathrm{~nm}$ was assessed using a microplate reader (Bio-Rad Laboratories, Inc.).

Wound healing assay. Transfected cells were seeded into 6 -well plates at a density of $5 \times 10^{5}$ cells/well and cultured to 
B

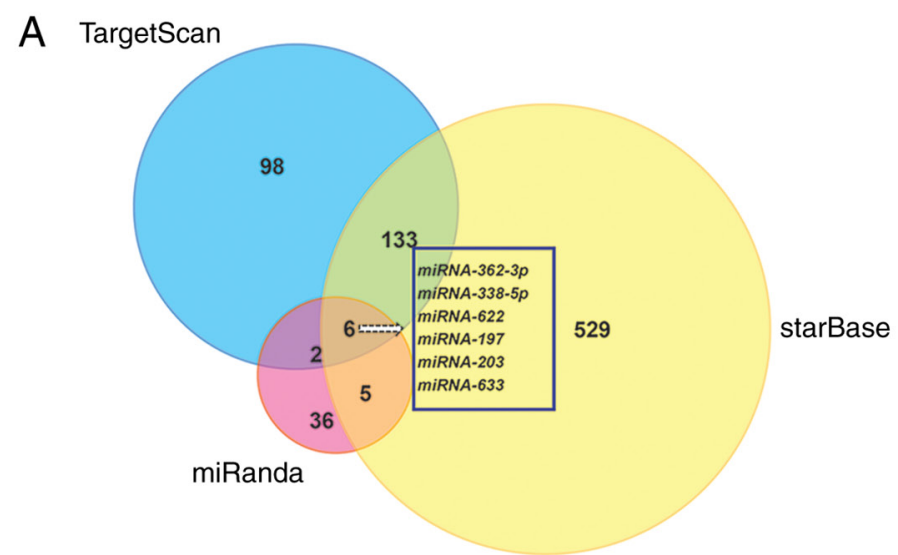

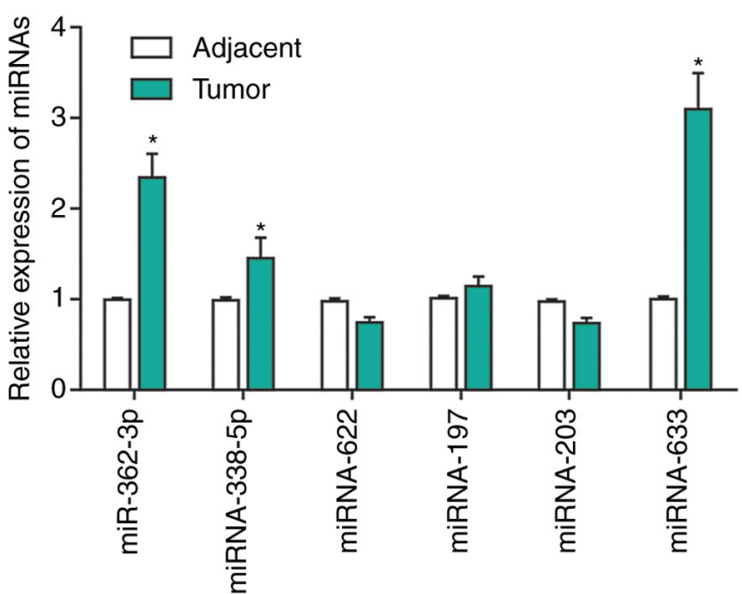

Figure 1. miRNAs predicted to regulate KAIl expression in melanoma. (A) Venn diagram of prediction results from the TargetScan, miRanda and StarBase analyses. Six miRNAs were predicted in common by all three analysis tools. (B) Reverse transcription-quantitative PCR results for the expression levels of the six miRNAs in melanoma tumor and adjacent normal tissues. "P<0.05. miRNA, miRNA; KAI1, CD82 antigen.

confluency in RPMI-1640 medium with $15 \%$ FBS at $37^{\circ} \mathrm{C}$ with $5 \% \mathrm{CO}_{2}$. A linear wound was made using a10 $\mu \mathrm{l}$ sterile pipette tip across the confluent cell layer, and the plates were washed twice to remove detached cells and debris. The cells were cultured in RPMI-1640 medium with $2.5 \%$ FBS, $24 \mathrm{~h}$ after the wound scraping. Then, the size of the wounds was observed and measured at 0 and $24 \mathrm{~h}$ under a light microscope (magnification, x20).

Invasion assay. To assess cell invasion, $3 \times 10^{4}$ cells were resuspended in serum-free RPMI-1640 medium (Gibco; Thermo Fisher Scientific, Inc.) and added to the upper portion of a Transwell chamber (Corning Inc.) that had been coated with Matrigel $^{\mathrm{TM}}$. At total of $600 \mu \mathrm{l}$ media containing 20\% FBS was added to the lower chamber, and plates were subsequently incubated for $24 \mathrm{~h}$. Cells that had invaded through the matrix to the lower chamber were subsequently fixed for $20 \mathrm{~min}$ using $4 \%$ paraformaldehyde, after which they were stained for $20 \mathrm{~min}$ with crystal violet. The cells were then observed using optical light microscopy (Olympus Corporation). The numbers of invaded cells in five random fields of view were subsequently quantified for each sample.

Western blotting. RIPA buffer (Beyotime Institute of Biotechnology) was used to lyse cells, after which a BCA kit (Beyotime Institute of Biotechnology) was used to quantify protein levels in the lysates. Protein (40 ug/lane) was subsequently subjected to electrophoretic separation (12\% SDS-PAGE), transferred to PVDF membranes (EMD Millipore), and blocked with 5\% non-fat milk at room temperature for $2 \mathrm{~h}$. The membranes were then incubated overnight with primary antibodies (cat. no. 10205-2-A; 1:1,000; ProteinTech Group, Inc.) at $4^{\circ} \mathrm{C}$. Blots were subsequently incubated at room temperature for $2 \mathrm{~h}$ with HRP-conjugated secondary antibodies (cat. no. KC-4G3; 1:20,000; Kang Chen Biotech, Inc.), after which ECL (Pierce; Thermo Fisher Scientific, Inc.) was used to detect the protein bands. Image $\mathrm{J}$ software $(1.48 \mathrm{u}$ version; National Institutes of Health) was used for densitometric analyses.
Statistical analysis. All statistical analyses were performed using SPSS 21.0 (IBM Corp.) and GraphPad Prism 7.0 (GraphPad Software, Inc.). Data are expressed as the mean \pm SD. Multiple group comparisons were analyzed using one-way analysis of variance and Tukey's post hoc test. Independent-samples t-tests were used to analyze the significance of mRNA levels in tumor tissues and adjacent normal tissues. Associations between the expression of pairs of genes were evaluated using Spearman's rank correlation analyses. Independent-samples t-tests were used to analyze the significance of cell proliferation, wound healing/migration and Transwell invasion assay results. All comparisons were two-tailed. $\mathrm{P}<0.05$ was considered to indicate a statistically significant difference.

\section{Results}

Identification of miRNAs that potentially target KAII in melanoma via bioinformatics prediction analyses. The potential upstream miRNAs that may target KAI1 were selected using the TargetScan, StarBase and miRanda online tools (27). In total, 6 putative regulators of KAI1 (miR-633, miR-362, miR-338, miR-622, miR-203 and miR-197) were identified using this approach (Fig. 1A). The expression levels of these miRNAs were subsequently measured in tumors from patients with melanoma and their matched adjacent normal tissue samples (Fig. 1B). Among the six miRNA candidates, miR-633 was found to be upregulated the most in melanoma tissues compared with adjacent normal tissues.

miR-633 is upregulated in melanoma and is negatively correlated with KAII expression. miR-633 was found to be expressed at higher levels in melanoma cells compared with HeMn control cells (Fig. 2A). Similarly, it was found that miR-633 was upregulated in melanoma tumor tissues compared with adjacent normal tissues, the results were re-plotted in Fig. 2B for ease of reference (Fig. 2B). When the mRNA expression levels for the KAI1 gene were examined in the same samples, the results exhibited an opposite pattern, 
A
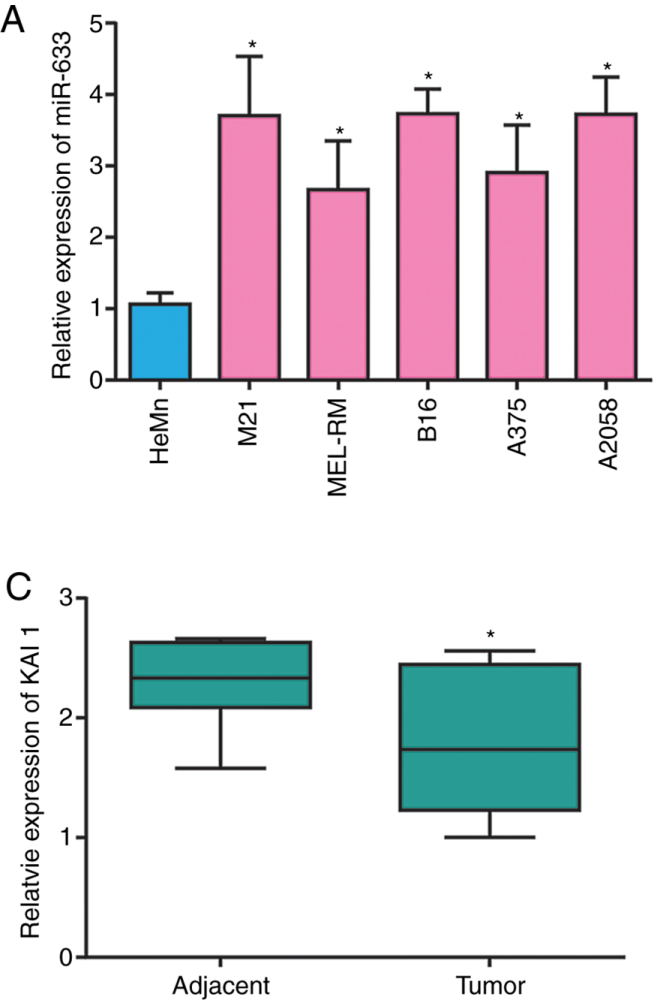

$\mathrm{B}$
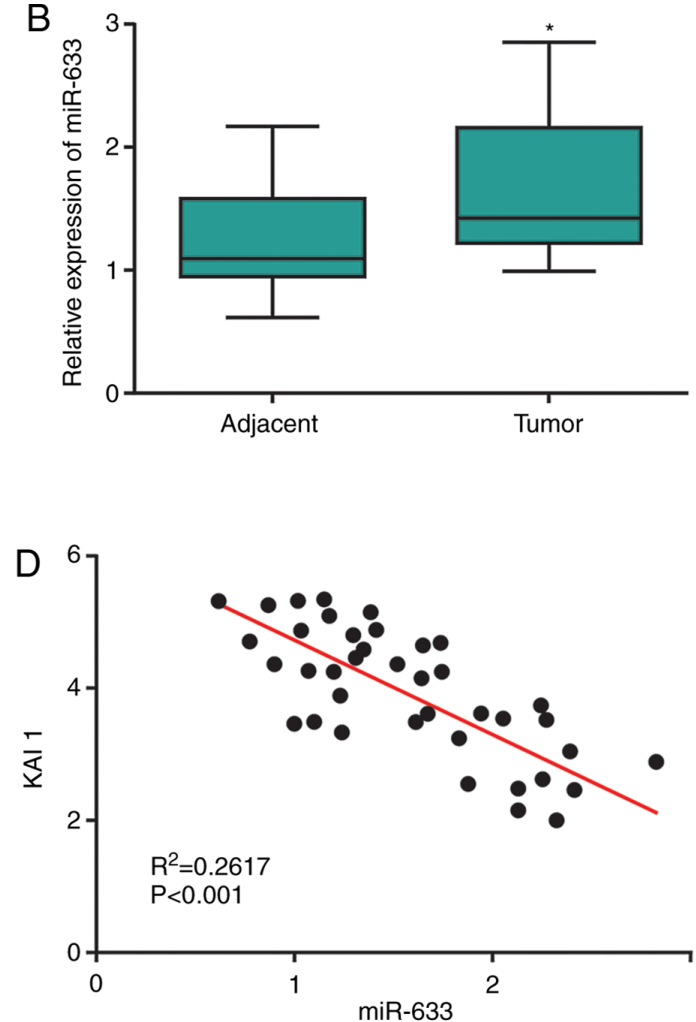

Figure 2. miR-633 is upregulated in melanoma and is negatively correlated with KAI1 expression. (A) The expression of miR-633 was assessed in HeMn and melanoma cell lines. (B) miR-633 expression levels and (C) KAI1 mRNA expression levels were evaluated in melanoma tumor and adjacent normal tissue samples from patients. (D) The expression of miR-633 was negatively correlated with that of KAI1 in melanoma tissues. ${ }^{*}<0.05$ vs. HeMn. miR, microRNA; KAI1, CD82 antigen.

with KAI1 mRNA expression levels significantly decreased in melanoma tissues compared with normal tissues (Fig. 2C). Pearson correlation analyses confirmed that miR-633 and KAI1 expression was negatively correlated in melanoma tissue samples (Fig. 2D).

miR-633 suppresses KAIl expression in melanoma. After having identified the predicted miR-633 binding site within the KAI1 3'-UTR, WT and MUT versions of a KAI1 luciferase reporter were constructed (Fig. 3A). Additionally, an miR-633 inhibitor and mimics were prepared, and their transfection efficiency was validated in A375 and B16 cells (Fig. 3B and $\mathrm{C}$ ). Using luciferase reporter assays, the results confirmed that miR-633 mimics were able to bind to the WT, but not the MUT, version of the KAI1 reporter, consistent with a specific binding interaction between miR-633 and KAI1 (Fig. 3D and E). Subsequently, it was determined that KAI1 expression was decreased at both the mRNA and the protein level in A375 and B16 cells overexpressing miR-633 (Fig. 3F and G). Taken together, these findings confirmed thatmiR-633 may serve as a negative regulator of KAI1 transcription and translation in melanoma cells.

miR-633 enhances melanoma cell proliferation and migration. Finally, the biological importance of miR-633 was assessed in melanoma. Using a CCK-8 assay, it was found that transfection with the miR-633 inhibitor significantly suppressed the numbers of melanoma cells over time compared with cells transfected with NC (Fig. 4A and B), suggesting a role for this miRNA in the proliferation of melanoma cells. In addition, miR-633 inhibitor transfection into both the A375 and B16 melanoma cell lines decreased migration rates in wound healing assays (Fig. 4C) and cell invasion in Transwell assays (Fig. 4D). Collectively, these findings demonstrated that miR-633 was able to promote the migration and proliferation of melanoma cells.

\section{Discussion}

In the present study, it was demonstrated that miR-633 was significantly upregulated in melanoma tissue compared with adjacent normal tissue from11patients with melanoma. In addition, a series of melanoma cell lines exhibited a significantly higher level of miR-633 expression compared with HeMn cells. In previous studies, miR-633 was shown to be a functionally important tumor-associated miRNA in lung and brain cancer $(28,29)$. Its functional importance in melanoma, however, had yet to be properly elucidated. The present study used the TargetScan, StarBase and miRanda algorithms to predict 6 candidate miRNAs that specifically targeted KAI1.KAI1 was shown to have one potential complementary miR-633-binding site within its 3'-UTR. Experimental results demonstrated that over expression of miR-633 led to a significant reduction in the levels of KAI1 protein, and deregulated expression of miR-633 significantly altered the proliferation, migration and invasion of A375 and B16 melanoma cells. Taken together, the findings of the present study have provided, to the best of the authors' knowledge, the first 
A KAl 13'-UTR Wt 5'-CCAGCCTTATGCTCGTGCAATTACG- $3^{\prime}$

B

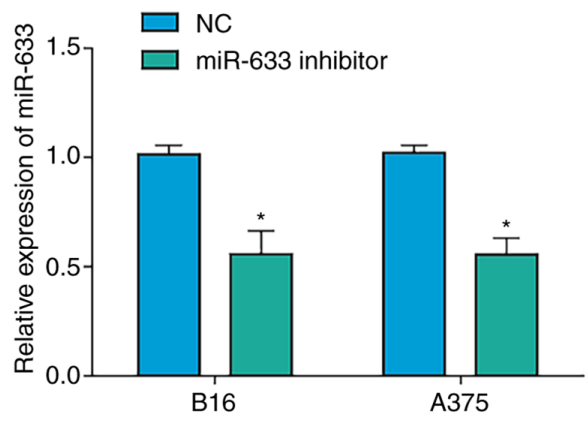

C

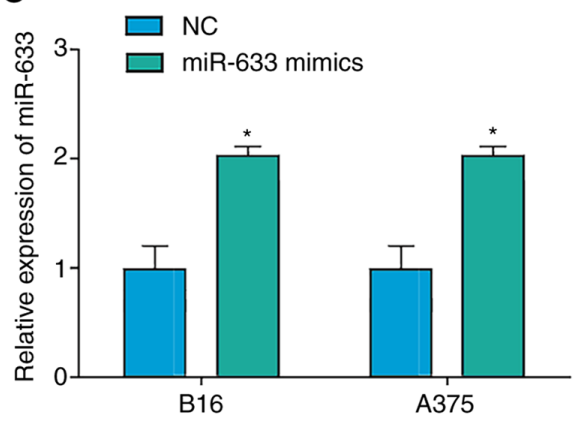

D

B16
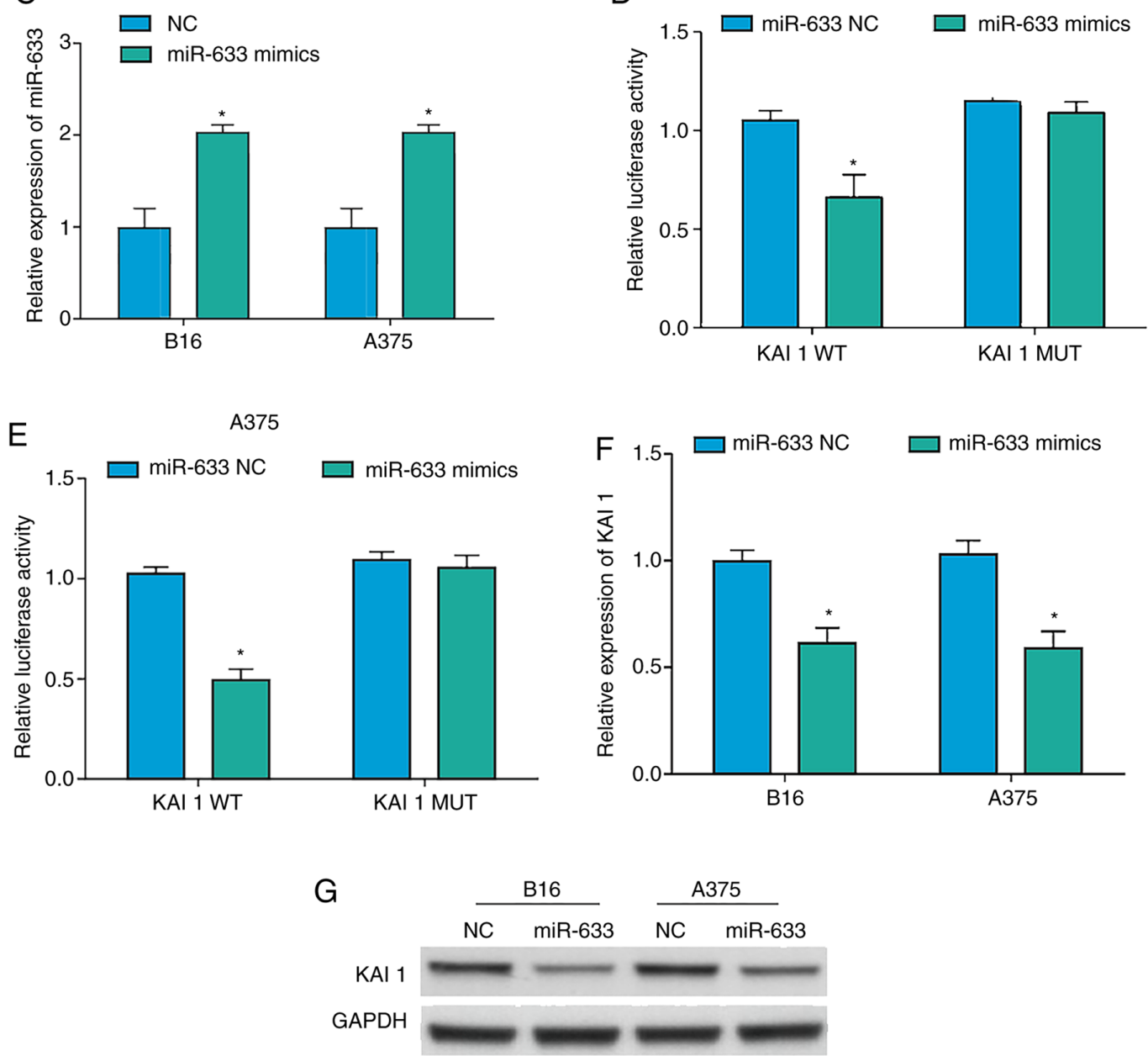

Figure 3. miR-633 suppresses KAI1 expression in melanoma cells. (A) The putative miR-633 binding site in the KAI1 3'-untranslated region. (B) miR-633 inhibition and (C) miR-633 overexpression were confirmed by reverse transcription-quantitative PCR in A375 and B16 cells, following transfection with miR-633 inhibitor or miR-633 mimics, respectively. (D) Luciferase activity was evaluated in B16 and (E) A375 cells, following co-transfection with KAI1 WT or KAI1 MUT constructs along with miR-633 mimics or controls. (F) KAI1 mRNA expression levels and (G) KAI1 protein expression levels were evaluated in B16 and A375 cells following miR-633 mimics or NC transfection. "P<0.05 vs. NC. miR, microRNA; KAI1, CD82 antigen; WT, wild type; MUT, mutant; NC, negative control.

evidence thatmiR-633 may function as a tumor promoter in human melanoma, mediated partly through targeting KAI1 expression.

A total of 3,657 mature human miRNAs have been identified to date (miRBase, 2019, http://www.mirbase.org). In prior studies, miRNAs identified from the integumentary system were successfully used as diagnostic and prognostic biomarkers in patients with melanoma $(30,31)$. For example, miR-213 is a suppressor of malignant melanoma progression, thereby indicating that it may be a potentially viable therapeutic target or diagnostic biomarker in patients with this form of cancer (32). Previous studies indicated that deregulation of miR-21 was associated with pro-apoptotic effects in pancreatic and breast cancer cell lines (33). In melanoma, a previous study revealed that miR-205, miR-200 and members of the let-7 family(-125b, -146a, -155 , $-21,-25,-23$ a and $-29 b$ ) were deregulated (34). Previous studies also suggested that abnormal miR-633 expression 
A

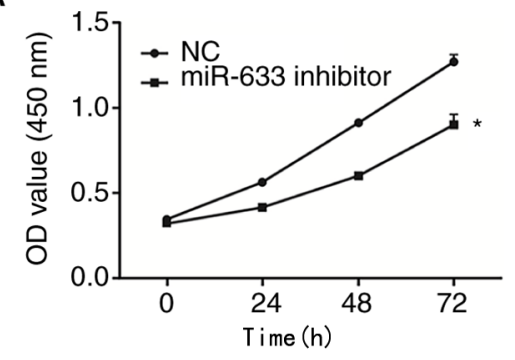

C
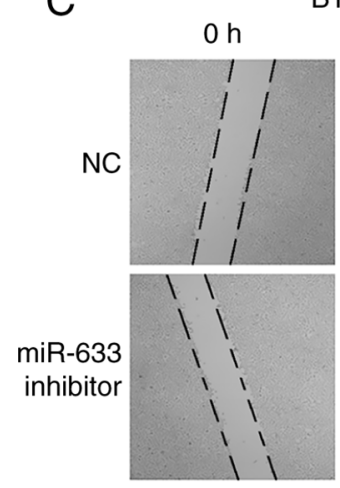
$24 \mathrm{~h}$
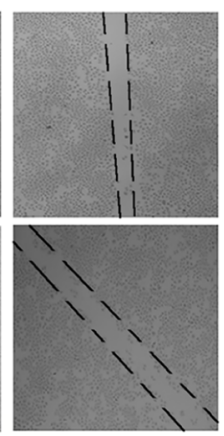

B16

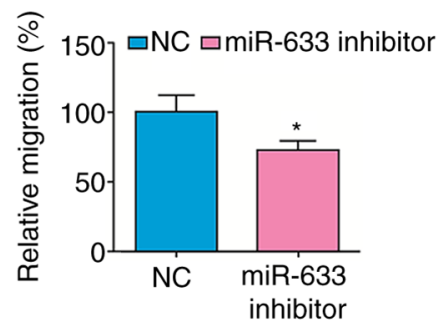

$0 \mathrm{~h}$

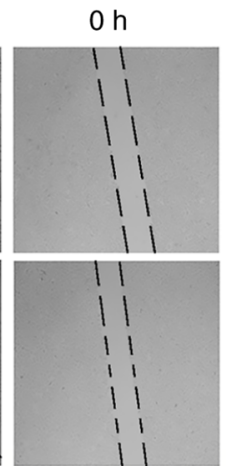

年1
A375

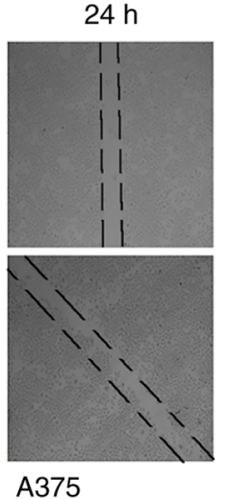

A375
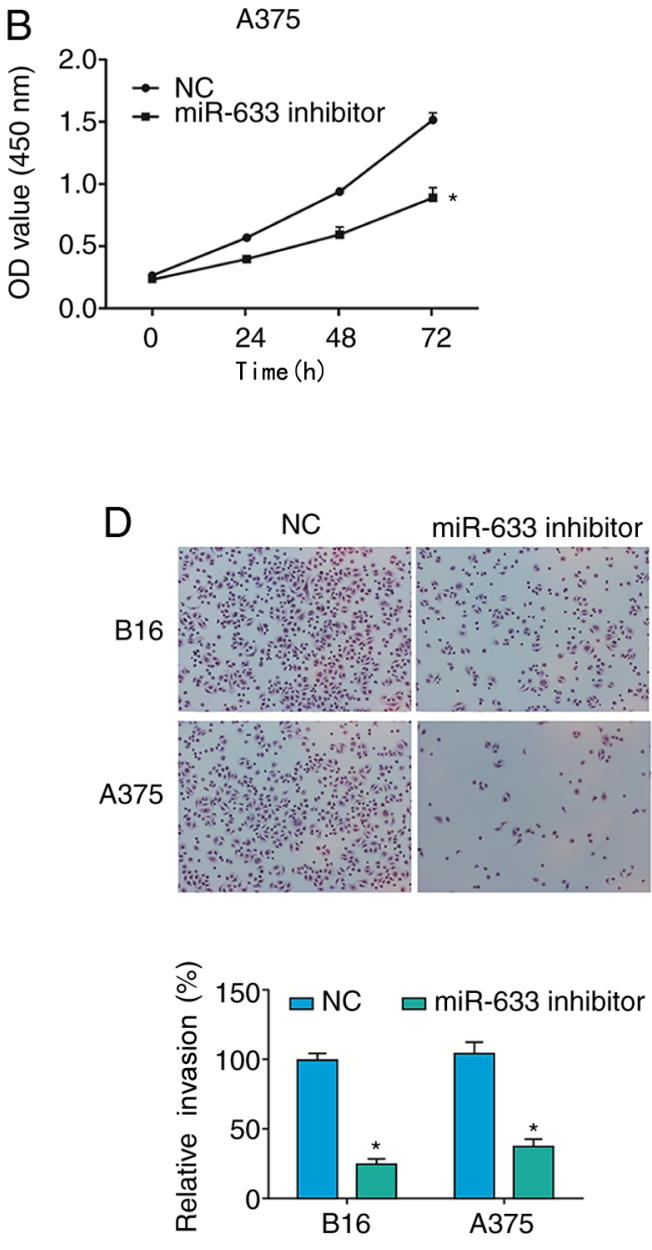

Figure 4. miR-633 regulates melanoma cell proliferation and migration. (A) The viability of B16 and (B) A375 cells was assessed following miR-633 inhibitor or NC transfection. (C) Wound healing activity was assessed for A375 and B16 cells following transfection with miR-633 inhibitor or NC (magnification, $\mathrm{x} 20$ ). (D) Transwell invasion activity was evaluated in A375 and B16 cells following miR-633 inhibitor or NC transfection (magnification, $\mathrm{x} 20$ ). $\mathrm{P}<0.05 \mathrm{vs.} \mathrm{NC.} \mathrm{miR,}$ microRNA; OD, optical density; NC, negative control.

was associated with the prednisone response early in childhood acute lymphoblastic leukemia relapse (35). However, abnormal miR-633 expression has only been definitively identified in few types of tumors to date. The association between miR-633 and malignant melanoma has not been previously elucidated. The present study demonstrated that the expression levels of miR-633 were upregulated in melanoma tissues and cell lines compared with normal tissues and cells, respectively. Furthermore, the results obtained revealed that deregulation of miR-633 significantly altered the proliferation and migration of A375 and B16 melanoma cells. Therefore, miR-633 may serve as a novel biomarker for melanoma in the future.

KAI1, also known as CD82, is an important transcriptional regulator and tumor suppressor gene, and KAI1 mutations are commonly encountered in a range of tumor types (36). KAI1 regulates chromatin accessibility, in part via the E-cadherin pathway (37), and prior bioinformatics analyses have suggested that KAI1 mutations are associated with lung, bone and brain cancer prognoses, underscoring the relevance of this gene in oncogenic contexts (38). KAI1 may attenuate signaling to shut down metastatic colonization through attenuation of epidermal growth factor receptor signaling and inhibition of the Wnt signaling pathway. You et al (39) suggested that the KAI1 promoter may be regulated by the p53 and NME/NM23 nucleoside diphosphate kinase 1 genes. In pancreatic carcinoma, over expressing KAI1 attenuated the phosphorylation of SRC and STAT3, thereby inhibiting the expression of vascular endothelial growth factor $\mathrm{C}$ and limiting the activity of pancreatic carcinoma cells (40). KAI1 has also been shown to serve as a mediator of metabolic reprogramming in tumor cells. For example, KAI1 can suppress the progression of cancers of the digestive system via influencing invasion-associated protein metabolism (41). It also serves as an important tumor suppressor gene in the context of integumental tumors, with KAI1 inactivating mutations being a common feature in human melanoma (42). The ability of KAI1 to suppress oncogenesis is linked to its ability to inhibit the expression of AP-1, JUNB, poly (ADP-ribose) polymerase (PARP) and other oncogenes (43). Khan et al (44) reported that KAI1 is a key regulator of Toll-like receptor 9 (TLR9) trafficking and signaling. KAI1 modulates TLR9-dependent NF- $\kappa \mathrm{B}$ nuclear translocation, which is critical for inflammatory cytokine production. A recent study demonstrated that KAI1 was significantly associated with poor survival and could act as 
an independent prognostic factor in human melanoma for both 5-year and 10-year survival rates (45). KAI1 deregulation has been observed in melanoma cell lines and tumor samples, and the over expression of this protein may significantly reduce melanoma progression (46). Furthermore, miR-203 inhibited frizzled-2 expression via KAI1 expression in human lung carcinoma cells, andKAIlexpression may be a useful marker for metastatic, invasive cancer, and prognostic factor in tumors, such as lung cancer (47). However, the specific mechanisms governing KAI1 downregulation in the context of melanoma had yet to be clarified.

The present study aimed to investigate the role of miR-633 in melanoma through its potential interaction with KAI1, revealing that miR-633 may be an important regulator of malignant melanoma. The results showed that miR-633 was upregulated in melanoma cells and tumor tissue samples compared with their controls. In addition, miR-633 inhibition resulted in impaired migration and proliferation of A375 and B16 melanoma cells in vitro. Notably, the results of the dual-luciferase reporter assay demonstrated that miR-633 directly regulated the expression of KAI1. Therefore, the data obtained in the present study have provided important insights into the regulation of KAI1 by miRNAs in cancer cells. miR-633 may serve as a potential candidate for the diagnosis and treatment of human melanoma.

In conclusion, the present study has provided novel evidence to show that inhibition of miR-633 suppresses the proliferation and migration of the malignant melanoma cell lines A375 and B16, partly via regulation of KAI1.

\section{Acknowledgements}

Not applicable.

\section{Funding}

No funding was received.

\section{Availability of data and materials}

All data generated or analyzed during the present study are included in this published article.

\section{Authors' contributions}

YL and ZW made substantial contributions to the conception and design of the study. Both authors collected the samples and clinical data, and contributed significantly to data analysis. ZW performed the experiments and drafted the initial manuscript. YL gave final approval of the version to be published. Both authors agreed to be accountable for all aspects of the work in ensuring that questions related to the accuracy and integrity of any part of the work were appropriately investigated and resolved. Both authors have read and approved the final manuscript.

\section{Ethics approval and consent to participate}

The present study was approved by the Ethics Committee of Cangzhou Central Hospital (Cangzhou, China; approval no. 201903501). Written informed consent was provided by patients and their families prior to the study start.

\section{Patient consent for publication}

Not applicable.

\section{Competing interests}

The authors declare that they have no competing interests.

\section{References}

1. Stark MS, Gray ES, Isaacs T, Chen FK, Millward M, McEvoy A, Zaenker P, Ziman M, Soyer HP, Glasson WJ, et al: A panel of circulating MicroRNAs detects uveal melanoma with high precision. Transl Vis Sci Technol 8: 12, 2019.

2. Xia Z, Yang C, Yang X, Wu S, Feng Z, Qu L, Chen X, Liu L and Ma Y: MiR-652 promotes proliferation and migration of uveal melanoma cells by targeting HOXA9. Med Sci Monit 25: 8722-8732, 2019.

3. Fumagalli MR, Lionetti MC, Zapperi S and La Porta C: Cross-Talk between circRNAs and mRNAs modulates MiRNA-mediated circuits and affects melanoma plasticity. Cancer Microenviron 12: 95-104, 2019.

4. Yang C, Xia Z, Zhu L, Li Y, Zheng Z, Liang J and Wu L: MicroRNA-139-5p modulates the growth and metastasis of malignant melanoma cells via the PI3K/AKT signaling pathway by binding to IGF1R. Cell Cycle 18: 3513-3524, 2019.

5. Sánchez-Sendra B, García-Giménez JL, González-Muñoz JF, Navarro L, Murgui A, Terrádez L, Pinazo I, Martin JM and Monteagudo C: Circulating miRNA expression analysis reveals new potential biomarkers for human cutaneous melanoma staging. J Eur Acad Dermatol Venereol 34: e126-e129, 2020.

6. Sun HW, Yang GL, Wang SN, Zhang YJ, Ding JX and Zhang XN: MicroRNA-92a regulates the development of cutaneous malignant melanoma by mediating FOXP1. Eur Rev Med Pharmacol Sci 23: 8991-8999, 2019.

7. Nakamura $\mathrm{K}$ and Okuyama R: Immunotherapy for advanced melanoma: Current knowledge and future directions. J Dermatol Sci 83: 87-94, 2016.

8. Reale E, Taverna D, Cantini L, Martignetti L, Osella M, De Pittà C, Virga F, Orso F and Caselle M: Investigating the epi-miRNome: Identification of epi-miRNAs using transfection experiments. Epigenomics 11: 1581-1599, 2019.

9. Afrang $\mathrm{N}$ and Honardoost $\mathrm{M}$ : Cell cycle regulatory markers in melanoma: New strategies in diagnosis and treatment. Med J Islam Repub Iran 33: 96, 2019.

10. Hämäläinen M, Teppo HR, Skarp S, Haapasaari KM, Porvari K, Vuopala K, Kietzmann T and Karihtala P: NRF1 and NRF2 mRNA and protein expression decrease early during melanoma carcinogenesis: An insight into survival and MicroRNAs. Oxid Med Cell Longev 2019: 2647068, 2019.

11. Rossi E, Schinzari G, Maiorano BA, Pagliara MM, Di Stefani A, Bria E, Peris K, Blasi MA and Tortora G: Conjunctival melanoma: Genetic and epigenetic insights of a distinct type of melanoma. Int J Mol Sci 20: 5447, 2019.

12. Chen L, Karisma VW, Liu H and Zhong L: MicroRNA-300: A transcellular mediator in exosome regulates melanoma progression. Front Oncol 9: 1005, 2019.

13. Jiang W, Hou L, Wei J, Du Y, Zhao Y, Deng X and Lin X: Hsa-MiR-217 inhibits the proliferation, migration, and invasion in non-small cell lung cancer cells via targeting SIRT1 and P53/KAI1 signaling. Balkan Med J 37: 208-214, 2020.

14. Zhang Q, Huang F, Yao Y, Wang J, Wei J, Wu Q, Xiang $\mathrm{S}$ and $\mathrm{Xu} \mathrm{L}$ : Interaction of transforming growth factor- $\beta$-smads/microRNA-362-3p/CD 82 mediated by M2 macrophages promotes the process of epithelial-mesenchymal transition in hepatocellular carcinoma cells. Cancer Sci 110: 2507-2519, 2019.

15. Habibzadeh P, Honarvar B, Silawi M, Bahramjahan S, Kazemi A, Faghihi MA and Lankarani K: Association between rs2303861 polymorphism in CD82 gene and non-alcoholic fatty liver disease: A preliminary case-control study. Croat Med J 60: 361-368, 2019. 
16. Asada H, Tomiyasu H, Uchikai T, Ishihara G, Goto-Koshino Y, Ohno $\mathrm{K}$ and Tsujimoto $\mathrm{H}$ : Comprehensive analysis of miRNA and protein profiles within exosomes derived from canine lymphoid tumour cell lines. PLoS One 14: e0208567, 2019.

17. Liu CJ, Yang JH, Huang FZ, Yang JH, Liu CP, Mao XH, Yi WM, Shen XB, Peng C, Chen MF, et al: The role of miR-99b in mediating hepatocellular carcinoma invasion and migration. Eur Rev Med Pharmacol Sci 24: 7909, 2020.

18. Long J, Luo J and Yin X: MiR-338-5p promotes the growth and metastasis of malignant melanoma cells via targeting CD82. Biomed Pharmacother 102: 1195-1202, 2018.

19. Lee MS, Lee J, Kim YM and Lee H: The metastasis suppressor CD82/KAI1 represses the TGF- $\beta 1$ and wnt signalings inducing epithelial-to-mesenchymal transition linked to invasiveness of prostate cancer cells. Prostate 79: 1400-1411, 2019.

20. Li W, Hu M, Wang C, Lu H, Chen F, Xu J, Shang Y, Wang F, Qin J, Yan Q, et al: A viral microRNA downregulates metastasis suppressor CD82 and induces cell invasion and angiogenesis by activating the c-met signaling. Oncogene 36: 5407-5420, 2017.

21. Nishioka C,Ikezoe T, Pan B, Xu K and Yokoyama A: MicroRNA-9 plays a role in interleukin-10-mediated expression of E-cadherin in acute myelogenous leukemia cells. Cancer Sci 108: 685-695, 2017.

22. Xu L, Hou Y, Tu G, Chen Y, Du YE, Zhang H, Wen S, Tang X, Yin J, Lang L, et al: Nuclear drosha enhances cell invasion via an EGFR-ERK1/2-MMP7 signaling pathway induced by dysregulated miRNA-622/197 and their targets LAMC2 and CD82 in gastric cancer. Cell Death Dis 8: e2642, 2017.

23. Jee BK, Park KM, Surendran S, Lee WK, Han CW, Kim YS and Lim Y: KAI1/CD82 suppresses tumor invasion by MMP9 inactivation via TIMP1 up-regulation in the H1299 human lung carcinoma cell line. Biochem Biophys Res Commun 342: 655-661, 2006

24. Mine M, Yamaguchi K, Sugiura T, Chigita S, Yoshihama N, Yoshihama R, Hiyake N, Kobayashi Y and Mori Y: MiR-203 inhibits frizzled-2 expression via CD82/KAI1 expression in human lung carcinoma cells. PLoS One 10: e0131350, 2015.

25. Zhang QH, Yao YL, Wu XY, Wu JH, Gu T, Chen L, Gu JH, Liu Y and $\mathrm{Xu}$ L: Anti-MiR-362-3p inhibits migration and invasion of human gastric cancer cells by its target CD82. Dig Dis Sci 60 1967-1976, 2015

26. Livak KJ and Schmittgen TD: Analysis of relative gene expression data using real-time quantitative PCR and the 2(-Delta Delta C(T)) method. Methods 25: 402-408, 2001

27. Dai W, Wang C, Wang F, Wang Y, Shen M, Chen K, Cheng P, Zhang Y, Yang J, Zhu R, et al: Anti-MiR-197 inhibits migration in HCC cells by targeting KAI 1/CD82. Biochem Biophys Res Commun 446: 541-548, 2014.

28. Li Q, Zhang LY, Wu S, Huang C, Liu J, Wang P and Cao Y: Bioinformatics analysis identifies MicroRNAs and target genes associated with prognosis in patients with melanoma. Med Sci Monit 25: 7784-7794, 2019.

29. Cooling L: An update on the I blood group system. Immunohematology 35: 85-90, 2019.

30. Santoni G, Morelli MB, Santoni M, Nabissi M, Marinelli O and Amantini C: Targeting transient receptor potential channels by microRNAs drives tumor development and progression. Adv Exp Med Biol 1131: 605-623, 2020

31. Ylösmäki L, Polini B, Carpi S, Martins B, Smertina E, Feola S, Fusciello M, Peltonen K, Nieri P, Ylösmäki E and Cerullo V: Harnessing therapeutic viruses as a delivery vehicle for RNA-based therapy. PLoS One 14: e0224072, 2019.

32. Sharma A, Biswas A, Liu H, Sen S, Paruchuri A, Katsonis P, Lichtarge O, Dakal TC, Maulik U, Gromiha MM, et al: Mutational landscape of the BAP1 locus reveals an intrinsic control to regulate the miRNA network and the binding of protein complexes in uveal melanoma. Cancers (Basel) 11: 1600, 2019.
33. Chekhun VF, Borikun TV, Bazas VM, Andriiv AV, Klyusov OM, Yalovenko TM and Lukianova NY: Association of circulating miR-21, -205, and -182 with response of luminal breast cancers to neoadjuvant FAC and AC treatment. Exp Oncol 42: 162-166, 2020.

34. Bonazzi VF, Stark MS and Hayward NK: MicroRNA regulation of melanoma progression. Melanoma Res 22: 101-113, 2012.

35. Xu L, Liang Yn, Luo Xq, Liu Xd and Guo Hx: Association of miRNAs expression profiles with prognosis and relapse in childhood acute lymphoblastic leukemia. Zhonghua Xue Ye Xue Za Zhi 32: 178-181, 2011 (In Chinese).

36. Wang Y, Chen H, Fu Y, Ai A, Xue S, Lyu O and Kuang Y: MiR-195 inhibits proliferation and growth and induces apoptosis of endometrial stromal cells by targeting FKN. Int J Clin Exp Pathol 6: 2824-2834, 2013.

37. Arribas AJ, Campos-Martín Y, Gómez-Abad C, Algara P, Sánchez-Beato M, Rodriguez-Pinilla MS, Montes-Moreno S, Martinez N, Alves-Ferreira J, Piris MA and Mollejo M: Nodal marginal zone lymphoma: Gene expression and miRNA profiling identify diagnostic markers and potential therapeutic targets. Blood 119: e9-e21, 2012.

38. Zhou X, Liu X, Zhang G, Zhang Q, Chen H, Wang Y, Fang F and Sun J: Knockdown THOC2 suppresses the proliferation and invasion of melanoma. Bioengineered 10: 635-645, 2019.

39. You J, Chang R, Liu B, Zu L and Zhou Q: Nm23-H1 was involved in regulation of KAI1 expression in high-metastatic lung cancer cells L9981. J Thorac Dis 8: 1217-1226, 2016.

40. Liu X, Guo X, Li H, Chen J and Qi X: Src/STAT3 signaling pathways are involved in KAI1-induced downregulation of VEGF-C expression in pancreatic cancer. Mol Med Rep 13: 4774-47778, 2016.

41. Bhalla S, Kaur H, Dhall A and Raghava GP: Prediction and analysis of skin cancer progression using genomics profiles of patients. Sci Rep 9: 15790, 2019.

42. Hou Q, Han S, Yang L, Chen S, Chen J, Ma N, Wang C, Tang J, Chen X, Chen F, et al: The interplay of microRNA-34a, LGR4, EMT-associated factors, and MMP2 in regulating uveal melanoma cells. Invest Ophthalmol Vis Sci 60: 4503-4510, 2019.

43. Lu T, Chen S, Qu L, Wang Y, Chen HD and He C: Identification of a five-miRNA signature predicting survival in cutaneous melanoma cancer patients. Peer J 7: e7831, 2019.

44. Khan NS, Lukason DP, Feliu M, Ward RA, Lord AK, Reedy JL, Ramirez-Ortiz ZG, Tam JM, Kasperkovitz PV, Negoro PE, et al: CD82 controls CpG-dependent TLR9 signaling. FASEB J 33: 12500-12514, 2019.

45. Zhang G, Cheng Y, Chen G, Tang Y, Ardekani G, Rotte A, Martinka M, McElwee K, Xu X, Wang Q and Zhou Y: Loss of tumor suppressors KAI1 and p27 identifies a unique subgroup of primary melanoma patients with poor prognosis. Oncotarget 6 : 23026-23035, 2015.

46. Liu N, Liu Z, Liu X and Chen H: Comprehensive analysis of a competing endogenous RNA network identifies seven-lncRNA signature as a prognostic biomarker for melanoma. Front Oncol 9: 935, 2019

47. Prabhu VV and Devaraj SN: KAI1/CD82, metastasis suppressor gene as a therapeutic target for non-small-cell lung carcinoma. J Environ PatholToxicol Oncol 36: 269-275, 2017.

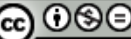

This work is licensed under a Creative Commons Attribution-NonCommercial-NoDerivatives 4.0 International (CC BY-NC-ND 4.0) License. 